\title{
Two-point active microrheology in a viscous medium exploiting a motional resonance excited in dual-trap optical tweezers
}

\author{
Shuvojit Paul, ${ }^{1}$ Randhir Kumar, ${ }^{1}$ and Ayan Banerjee ${ }^{1, *}$ \\ ${ }^{1}$ Indian Institute of Science Education and Research, Kolkata, India
}

(Dated: January 22, 2018)

\begin{abstract}
Two-point microrheology measurements from widely separated colloidal particles approach the bulk viscosity of the host medium more reliably than corresponding single point measurements. In addition, active microrheology offers the advantage of enhanced signal to noise over passive techniques. Recently, we reported the observation of a motional resonance induced in a probe particle in dual-trap optical tweezers when the control particle was driven externally [Paul et al. Phys. Rev. E 96, 050102(R) (2017)]. We now demonstrate that the amplitude and phase characteristics of the motional resonance can be used as a sensitive tool for active two-point microrheology to measure the viscosity of a viscous fluid. Thus, we measure the viscosity of viscous liquids from both the amplitude and phase response of the resonance, and demonstrate that the zero-crossing of the phase response of the probe particle with respect to the external drive is superior compared to the amplitude response in measuring viscosity at large particle separations. We compare our viscosity measurements with that using a commercial rheometer and obtain an agreement $\sim 1 \%$. The method can be extended to viscoelastic material where the frequency dependence of the resonance may provide further accuracy for active microrheological measurements.
\end{abstract}

\section{INTRODUCTION}

Microrheology measurements have enabled the measurement of viscosity using trace volumes of liquids and have proved to be especially useful in the context of biology, where the rheology of cellular environments naturally involve extremely small sample volumes [1 $[3]$. Microrheology is performed using Brownian tracer probes, and can be performed using both 'passive' and 'active' methods. In the former, the thermal motion of the Brownian probes embedded and diffusing freely in the medium is measured [4 9] while in the latter, an external force is applied to the probe(s) via optical or magnetic tweezers, and the response determined [10 15]. It is straightforward to comprehend that active microrheology provides greater signal-to-noise in measurements compared to the passive method, since thermal motion of the probes is manifested by the auto or cross-correlation amplitudes of their Brownian displacements which are often weak and therefore limited by experimental noise. In contrast, active microrheology is performed by an external forcing of the probe with amplitudes much greater than that of the inherent Browninan flucturations, so that the corresponding response is also much larger [12, 13].

However, both active and passive microrheology measurements mostly use a single probe particle embedded in the medium. This is known as one-point microrheology, which has been implemented by both passive and active techniques, as mentioned earlier. Optical traps - with their ability to provide long measurement times are ideal for microrheology applications so that they are also often used useful for passive microrheology [3, 12, 13, 16, 19]. In addition, they allow the possibility of detection over a large frequency bandwidth with the use of position sensitive photodetectors that have much larger bandwidth compared to standard video microscopy that is typically preferred for freely diffusing probes. It must be noted, though, that measurements of the local viscosity using a single probe implies that these are over a very localized region of the sample, which may not necessarily represent the bulk viscosity very accurately. This may be due to the presence of inhomogeneities in temperature or density the latter being particularly relevant for complex or viscoelastic material. In addition, determining rheological parameters from one-point microrheology by employing optical tweezers may be erroneous since the high intensity of the tweezers laser may lead to local heating and an enhanced temperature of the liquid in the immediate vicinity of the probe [20]. Thus, two-point microrheology often provides more reliable estimation of the bulk viscosity/viscoelasticity as has been pointed out in the literature $[21+25]$. Two point passive microrheology using optical tweezers has also been performed [26, but instances of active two-point microrheology using tweezers are rare. It is important to note that in the case of passive microrheology - the hydrodynamic interaction between two Brownian probes decreases inversely [27, 28. with their separation and hence it is challenging to experimentally measure such cross-correlations with large signal to noise when the inter-particle distance is large. Thus, it is preferable to develop optical tweezers-based active two-point microrheology techniques in order to improve the efficacy of such measurements.

In this paper, we describe a two-point active microrheology approach using dual trap optical tweezers, where the trapped particles are widely separated - thus yielding viscosity values that are very close to the bulk viscosity of a viscous fluid. In our method, we drive one of the trapped particles in the dual trap sinusoidally, and exploit the fact that hydrodynamic interactions between 
the particles leads to a motional resonance in the driven particle at a particular drive frequency. Thus, we measure the resonance frequency from both the maximum amplitude of the driven particle and the zero-crossing of its phase with respect to the drive frequency. The value of this resonance frequency is dependent on the medium viscosity - all other factors (such as trap stiffnesses and particle separation) being unchanged - so that any shifts will correspond to changes in viscosity of the fluid medium. We validate the technique in water where we compare our measured values of viscosity with the standard value for water. We also measure the accuracy of the technique at different particle separations and find that the the zero crossing of the phase provides more accurate results. We then proceed to determine the viscosity of a water and glycerol solution with different concentrations of glycerol. Our measured viscosity values agree with that obtained using a commercial rheometer at the level of $\sim 1 \%$. Our method is naturally extendable to viscoelastic or even active material where the effect of retarded hydrodynamic interactions as well the dispersion in viscosity could lead to more intriguing characteristics of the resonance which may increase the accuracy as well as sensitivity of active two-point microrheology.

\section{THEORETICAL ANALYSIS}

The detailed theoretical treatment for the amplitude and phase response of the driving (control) and driven (probe) particles in two hydrodynamically coupled optical traps has been given in [29. Here, we provide a brief description for completeness. The Langevin equations describing the stochastic trajectories of the trapped particles are given by 30 .

$$
\begin{aligned}
m_{i} \dot{\mathbf{v}}_{i} & =-\gamma_{i j} \mathbf{v}_{j}-\nabla_{i} U+\boldsymbol{\xi}_{i} \\
\dot{\mathbf{R}}_{i} & =\mathbf{v}_{i}
\end{aligned}
$$

where $i, j=1,2$ refer to the driving and driven particle, $m_{i}$ are their masses, $\boldsymbol{v}_{i}$ are their velocities, $\gamma_{i j}$ are the second-rank friction tensors encoding the velocity-dependent dissipative forces mediated by the fluid, $U=U_{1}+U_{2}$ is the total potential of the conservative forces, and $\boldsymbol{\xi}_{i}$, the Langevin noises, are zero-mean Gaussian random variables whose variance is provided by the fluctuation-dissipation relation $\left\langle\boldsymbol{\xi}_{i}(t) \boldsymbol{\xi}_{j}\left(t^{\prime}\right)\right\rangle=$ $2 k_{B} T \gamma_{i j} \delta\left(t-t^{\prime}\right)$. The bold-face notation, with Cartesian indices suppressed, is used for both vectors and tensors in the above Eq. $\dot{\mathbf{R}}_{i}$ and $\dot{\mathbf{v}}_{i}$ are position and velocity of the $\mathrm{i}$-th particle, respectively. The optical potential is given by $U(t)=\frac{1}{2} \sum k_{i}\left(\mathbf{R}_{i}-\mathbf{R}_{i}^{0}\right)^{2}$ where $\mathbf{R}_{i}^{0}$ is the position of the potential minimum of the i-th optical trap and $k_{i}$ is the corresponding stiffness. In the experimental setup, the minimum of one of the optical trap (driving particle) is shifted with an external periodic signal and the re- sponse of the particle in the other trap (driven particle) is studied. Assuming momentum to be rapidly relaxing on the time scale of the trap motion, we neglect inertia and average over the noise to get,

$$
-\gamma_{i j} \dot{\mathbf{R}}_{j}-\nabla_{i} U=\mathbf{0}
$$

Eq 3 can be inverted and arranged to be represented in terms of mobility matrices $\boldsymbol{\mu}$ in the following manner (considering components of the matrix, where $\boldsymbol{\delta}$ is the $3 \times 3$ identity matrix),

$$
\begin{aligned}
& \dot{\mathbf{R}}_{1}=-\mu_{11} \boldsymbol{\delta} k_{1}\left(\mathbf{R}_{1}-\mathbf{R}_{1}^{0}\right)-\boldsymbol{\mu}_{12} k_{2}\left(\mathbf{R}_{2}-\mathbf{R}_{2}^{0}\right) \\
& \dot{\mathbf{R}}_{2}=-\boldsymbol{\mu}_{21} k_{1}\left(\mathbf{R}_{1}-\mathbf{R}_{1}^{0}\right)-\mu_{22} \boldsymbol{\delta} k_{2}\left(\mathbf{R}_{2}-\mathbf{R}_{2}^{0}\right)
\end{aligned}
$$

Thus, incorporating the mobility matrices with separation vector as the average distance between two the minima of the optical traps we have, with $k_{i} \mathbf{R}_{\mathrm{i}}^{\mathbf{0}}=\mathbf{F}_{\mathrm{i}}^{\mathbf{0}}$,

$$
\frac{d}{d t}\left[\begin{array}{l}
\mathbf{R}_{1} \\
\mathbf{R}_{2}
\end{array}\right]=-\left[\begin{array}{cc}
\mu_{11} k_{1} \boldsymbol{\delta} & \boldsymbol{\mu}_{12} k_{2} \\
\boldsymbol{\mu}_{21} k_{1} & \mu_{22} k_{2} \boldsymbol{\delta}
\end{array}\right]\left[\begin{array}{l}
\mathbf{R}_{1} \\
\mathbf{R}_{2}
\end{array}\right]+\left[\begin{array}{cc}
\mu_{11} \boldsymbol{\delta} & \boldsymbol{\mu}_{12} \\
\boldsymbol{\mu}_{21} & \mu_{22} \boldsymbol{\delta}
\end{array}\right]\left[\begin{array}{l}
\mathbf{F}_{1}^{0} \\
\mathbf{F}_{2}^{0}
\end{array}\right]
$$

The steady state solution of Eq4 can easily be calculated by working in the frequency domain. Thus, assum$\operatorname{ing} \mathbf{A}=\left[\begin{array}{cc}\mu_{11} k_{1} \boldsymbol{\delta} & \boldsymbol{\mu}_{12} k_{2} \\ \boldsymbol{\mu}_{21} k_{1} & \mu_{22} k_{2} \boldsymbol{\delta}\end{array}\right]$ and $\mathbf{M}=\left[\begin{array}{cc}\mu_{11} \boldsymbol{\delta} & \boldsymbol{\mu}_{12} \\ \boldsymbol{\mu}_{21} & \mu_{22} \boldsymbol{\delta}\end{array}\right]$, and taking Fourier transforms, we obtain,

$$
\mathbf{R}_{i}(\omega)=\left[[-i \omega \boldsymbol{\delta}+\mathbf{A}]^{-1} \mathbf{M}\right]_{i j} \mathbf{F}_{j}^{0}(\omega)=\chi_{i j}(\omega) \mathbf{F}_{j}^{0}(\omega)
$$

where we now introduce the response $\chi$, since one of the optical traps is modulated by a sinusoidal signal of driving frequency $\Omega$, which in the frequency domain may be represented as $\mathbf{F}_{j}^{0}(\omega)=\frac{X_{j}}{2}(\delta(\omega-\Omega)+\delta(\omega+\Omega))$, with $X_{j}$ being the amplitude of the drive. Further, $\chi$ is a block-diagonal matrix in cartesian indices. Given the experimental set up, $\boldsymbol{\chi}$ can be decomposed into $\boldsymbol{\chi}_{\|}$and $\chi_{\perp}$ for motion along the trap separation and the motion perpendicular to it. Inserting this form of the response into Eq. 7 and going back into the time domain, we have

$$
R_{\| i}(t)=\chi_{\| i j}^{\prime} \cos (\Omega t) X_{j}+\chi^{\prime \| i j} \sin (\Omega t) X_{j}
$$

Two time scales from two traps can be calculated to be $\tau_{i}=\frac{1}{\mu k_{i}}$, so that,

$$
\begin{array}{r}
\chi_{\|}(\Omega)=\frac{1}{\operatorname{Det} \boldsymbol{A}_{\|}-\Omega^{2}-i \Omega \operatorname{Tr} \boldsymbol{A}_{\|}} \times \\
{\left[\begin{array}{cc}
\left(\mu_{11} k_{1}-i \Omega\right) & \mu_{12} k_{2} \\
\mu_{21} k_{1} & \left(\mu_{22} k_{2}-i \Omega\right)
\end{array}\right]^{-1}\left[\begin{array}{ll}
\mu_{11} & \mu_{12} \\
\mu_{21} & \mu_{22}
\end{array}\right]} \\
=\frac{1}{\operatorname{Det} \boldsymbol{A}_{\|}-\Omega^{2}-i \Omega \operatorname{Tr} \boldsymbol{A}_{\|}} \times \\
{\left[\begin{array}{cc}
\left(\mu_{22} k_{2}-i \Omega\right) & -\mu_{12} k_{2} \\
-\mu_{21} k_{1} & \left(\mu_{11} k_{1}-i \Omega\right)
\end{array}\right]\left[\begin{array}{ll}
\mu_{11} & \mu_{12} \\
\mu_{21} & \mu_{22}
\end{array}\right]}
\end{array}
$$




$$
\begin{array}{r}
\chi_{\|}(\Omega)=\frac{\operatorname{Det} \boldsymbol{A}_{\|}-\Omega^{2}+i \Omega \operatorname{Tr} \boldsymbol{A}_{\|}}{\left(\operatorname{Det} \boldsymbol{A}_{\|}-\Omega^{2}\right)^{2}+\Omega^{2}\left(\operatorname{Tr} \boldsymbol{A}_{\|}\right)^{2}} \times \\
{\left[\begin{array}{cc}
k_{2} \operatorname{Det} \boldsymbol{M}_{\|}-i \mu \Omega & -i \Omega \mu_{12} \\
-i \Omega \mu_{21} & k_{1} \operatorname{Det} \boldsymbol{M}_{\|}-i \mu \Omega
\end{array}\right]}
\end{array}
$$

We are interested in the resonance in amplitude of the probe due to the forcing of the control, which is obtained by maximizing the modulus of $\chi_{\|_{21}}$ with respect to $\Omega$. Thus,

$$
\begin{aligned}
\left|\chi_{\|_{21}}\right| & =\left|\frac{-i \Omega \mu_{21}}{\operatorname{Det} \boldsymbol{A}_{\|}-\Omega^{2}-i \Omega \operatorname{Tr} \boldsymbol{A}_{\|}}\right| \\
& =\frac{\Omega \mu_{21}}{\sqrt{\left(\operatorname{Det} \boldsymbol{A}_{\|}-\Omega^{2}\right)^{2}+\Omega^{2}\left(\operatorname{Tr} \boldsymbol{A}_{\|}\right)^{2}}}
\end{aligned}
$$

and finally, the amplitude of the probe is given by

$$
A=\left|\chi_{\|_{21}}\right| k_{1} X=\frac{\Omega X \mu_{21} k_{1} X}{\sqrt{\left(\operatorname{Det} \boldsymbol{A}_{\|}-\Omega^{2}\right)^{2}+\Omega^{2}\left(\operatorname{Tr} \boldsymbol{A}_{\|}\right)^{2}}}
$$

, where $X$ is the amplitude of the driving signal on the control particle. The phase response of the probe with respect to the drive is given by

$$
\Delta \phi=\tan ^{-1}\left(\frac{\Omega^{2}-\operatorname{Det} \boldsymbol{A}_{\|}}{\Omega\left(\operatorname{Tr} \boldsymbol{A}_{\|}\right)}\right)
$$

Clearly the resonance frequency in dimensionless unit is $\tau_{1} \Omega_{r e s}=\sqrt{\frac{\operatorname{Det} \boldsymbol{A}_{\|}}{\mu_{11}^{2} k_{1}^{2}}}=\sqrt{\frac{k_{2}}{k_{1}}\left(1-\frac{\mu_{12}^{2}}{\mu_{11}^{2}}\right)}$ when $\mu_{12} \neq 0$.

Note that Eq. 10 is analogous to the expression for displacement of a forced harmonic oscillator, with the resonance frequency in this case given by $\omega_{0}^{2}=\operatorname{Det} \boldsymbol{A}_{\|}$ and the damping term $\Gamma=\operatorname{Tr} \boldsymbol{A}_{\|}$. Finally, we have

$$
\begin{gathered}
\omega_{0}^{2}=\operatorname{Det}_{\|}=k_{1} k_{2}\left(\mu_{11} \mu_{22}-\mu_{12} \mu_{21}\right) \\
\Gamma=\operatorname{Tr} \boldsymbol{A}_{\|}=\left(k_{1} \mu_{11}+k_{2} \mu_{22}\right)
\end{gathered}
$$

with $\mu_{i j}$ is the component of $\boldsymbol{\mu}_{i j}$ that is parallel to $\left(\mathbf{R}_{\mathbf{1}}^{\mathbf{0}}-\mathbf{R}_{\mathbf{2}}^{\mathbf{0}}\right)$. Here, $\mu_{11}=\mu_{22}=\frac{1}{6 \pi \eta a}, \mu_{12}=\mu_{21}=$ $\frac{1}{8 \pi \eta r_{0}}\left(2-\frac{4 a_{0}^{2}}{3 r_{0}^{2}}\right), a_{0}$ is the radius of the spherical particle, and $r_{0}$ is the trap separation.

An inspection of Eq. 13 reveals that $\omega_{0}$ is dependent on the stiffness $k_{1}, k_{2}$ and of the viscosity $\eta$ of the medium, which implies that for constant stiffness, any change in $\eta$ would be revealed in shifts in $\omega_{0}$. We now perform a set of theoretical simulations to find out the implications of these equations in the determination of viscosity of the ambient liquid where the particles are embedded. First, we study the change in $\omega_{0}$ with changing $\eta$ for different stiffness ratios of $k_{1}$ and $k_{2}$. Thus, we keep the value of $k_{2}$ constant $\left(k_{2}=3 \mu N / m\right.$, similar to what we use in
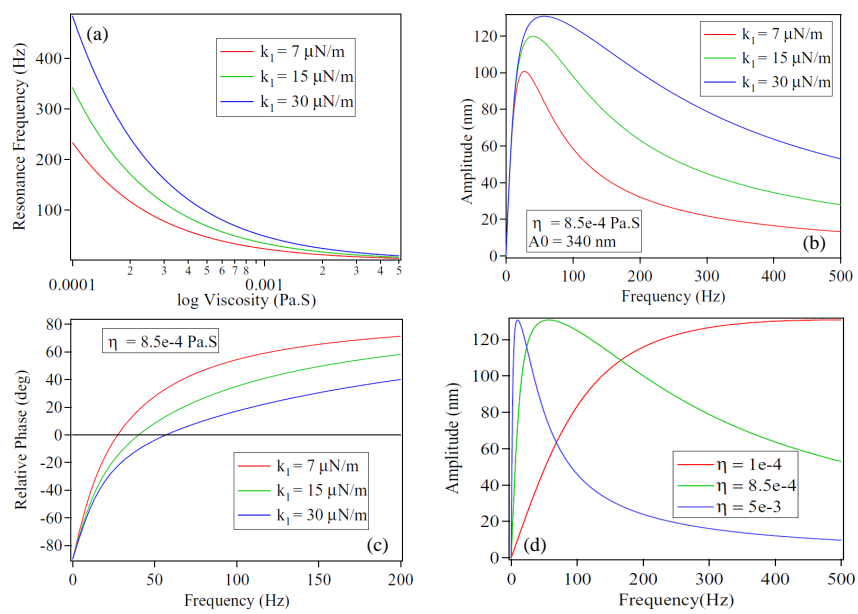

Figure 1. (a) Resonance frequency as a function of viscosity for different stiffnesses. $k_{2}$ is kept constant (at $3 \mu N / m$ ), while $k_{1}$ is varied - the red, green, and blue lines signifying $k_{1}$ values of 7,15 , and $30 \mu N / m$, respectively. (b) Amplitude of probe as a function of drive frequency for same stiffness values and colour code as (a). (c) Phase of probe as a function of drive frequency. (d) Amplitude of probe as a function of drive frequency for stiffness $k_{2}=3 \mu N / m, k_{1}=7 \mu N / m$ and different viscosity $(\eta)$ values of $1 \mathrm{e}-4$ (red), 8.5 e-4 (green), and 5 e-3 (blue).

experiments as described later), and use different values of $\left(k_{1}=7,15,30 \mu \mathrm{N} / \mathrm{m}\right)$ to find out how $\omega_{0}$ varies with changing $\eta$ over a range 1 e- 4 - 5 e- 3 Pa-s. We choose this range to mimic experimentally feasible conditions, noting that optical trapping is rendered rather difficult in liquids with viscosity lesser or larger than water by around an order of magnitude (viscosity of water being $8.5 \mathrm{e}-4 \mathrm{~Pa}-\mathrm{S}$ at $300 \mathrm{~K})$. This is shown in Fig. 1(a). It is clear that $\omega_{0}$ changes the most for the largest ratio of trap stiffnesses, i.e. when the product is largest (as is clear from Eq,13). However, it is incorrect to conclude that the experimental sensitivity in measuring $\eta$ is also highest for the highest stiffness ratio. Note that the measurement sensitivity would not only be dependent on shift in $\omega_{0}$, but also on how sensitively the shift can be measured from the amplitude and phase characteristics of the resonance. This essentially translates to the width of the resonance in terms of amplitude, and the change in phase across the resonance frequency. Thus, we study the the amplitude and phase response of the resonance for the same ratio of stiffnesses, as shown in Fig. 1(b) and (c). It is very clear that the width of the resonance increases with increasing stiffness ratio, so that the narrowest width is obtained for $k_{1}=7 \mu N / m$ for $k_{2}=3 \mu N / m$ (Fig. 1(b)). The maximum change of phase also occurs for the same combination of stiffness as shown in Fig. 11(c). Interestingly, the resonance peak-width reduces with increasing viscosity, as we observe in Fig. 1(d), where we plot the amplitude for three different viscosities $(\eta=1,8.5$, and 50 e-4 Pa.s) as shown in the figure. It is therefore clear that 

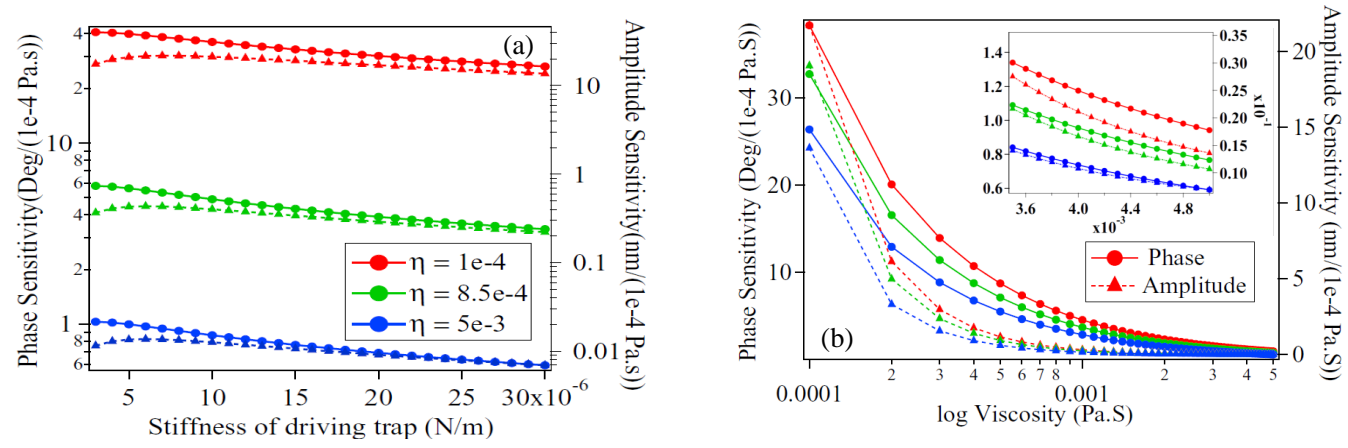

Figure 2. Phase (left axis, filled solid circles) and amplitude (right axis, filled solid triangles) sensitivities as a function of (a) $k_{1}$ and (b) (log) viscosity. Once again, $k_{2}$ is kept constant (at $\left.3 \mu N / m\right)$, while $k_{1}$ is varied - the red, green, and blue lines signifying $k_{1}$ values of 7,15 , and $30 \mu N / m$, respectively. In the inset, a zoomed-in version of the phase and amplitude sensitivities for large viscosity values is shown.

we have several different possibly competing effects that would finally decide the sensitivity for viscosity measurement. In order to obtain a quantitative understanding of this, we combine the shifts in frequency $\left(\Delta \omega_{0}\right)$ and that in resonance peak-width/phase change so as to write down the following relations for the phase $\left(\Phi_{s}\right)$ and amplitude sensitivities $\left(A_{s}\right)$ as:

$$
\begin{gathered}
\Delta \omega_{0}=\frac{\partial \omega_{0}}{\partial \eta} \Delta \eta=\frac{\eta \omega_{0} \Delta \eta}{\eta^{2}-\left(\frac{\Delta \eta}{2}\right)^{2}} \\
\Phi_{s}=\Phi\left(\omega_{0}+\Delta \omega_{0}\right)-\Phi\left(\omega_{0}\right)=\tan ^{-1}\left(\frac{\Delta \omega_{0}\left(2 \omega_{0}+\Delta \omega_{0}\right)}{\Gamma\left(\omega+\Delta \omega_{0}\right)}\right)
\end{gathered}
$$

$$
\begin{gathered}
A_{s}=A\left(\omega_{0}+\Delta \omega_{0}\right)-A\left(\omega_{0}\right)= \\
\frac{\mu_{21} k_{1} X\left(\omega_{0}+\Delta \omega_{0}\right)}{\sqrt{\left(\left(\omega_{0}+\Delta \omega_{0}\right)^{2}-\omega_{0}^{2}\right)^{2}+\left(\left(\omega_{0}+\Delta \omega_{0}\right) \Gamma\right)^{2}}}-\frac{\mu_{21} k_{1} X}{\Gamma}
\end{gathered}
$$

where, $\Delta \omega_{0}$ is the shift in resonance frequency due to $\Delta \eta$ change in viscosity around the viscosity $\eta . \Phi_{s}$ and $A_{s}$ are defined as the phase and amplitude sensitivity,

We now consider a situation where we continuously change the value of viscosity of the surrounding liquid, something which may arise in a practical situation for online monitoring of the viscosity of a liquid in situ. We use the three different stiffness ratios we had used earlier in Figs. 1(a)-(c). Once again, the phase sensitivity is indeed higher than the amplitude sensitivity. Following the trend observed in Fig. 2(a), both $A_{s}$ and $\Phi_{s}$ are respectively, and represent the changes in amplitude and phase due to change in viscosity $\Delta \eta$ at a particular resonance frequency, keeping all other parameters same.

It is now necessary to study $A_{s}$ and $\Phi_{s}$ carefully in order to obtain a complete understanding of the efficacy of our technique in measuring viscosity. Thus, we first proceed to plot $A_{s}$ and $\Phi_{s}$ against increasing $k_{1}$, keeping $k_{2}$ fixed at $3 \mu \mathrm{N} / \mathrm{m}$, at 3 different fixed values of viscosity of the surrounding liquid (same as Fig. 2(b)). This is shown in the right axis of the plot depicted in Fig[2(a). We observe that $A_{s}$ does not change substantially when we vary the stiffness of the driving trap $\left(k_{1}\right)$, so that it is clear that the increase in resonance frequency shift and the corresponding increase in peak-width almost compensate each other. For $A_{s}$, we have a maximum sensitivity around $k_{1}=7 \mu \mathrm{N} / \mathrm{m}$. However, the change is only about $25 \%$ in the entire range of $k_{1}$. In addition, the sensitivity reduces monotonically for increasing viscosity, so that we have the lowest sensitivity for the highest viscosity. This behaviour is also seen in $\Phi_{s}$ (left axis of the same figure). We notice that $\Phi_{s}$, unlike $A_{s}$, decreases linearly with increasing $k_{1}$, but the change is also only around $35 \%$ over the entire range. It is also clear that the value of the phase sensitivity is higher than the amplitude (tens of degrees, as compared to few $\mathrm{nm}$ ), which makes the phase measurement less challenging for experiments.

clearly dependent on the viscosity value being measured, with the sensitivity decreasing with increasing viscosity, though $\Phi_{s}$ changes less drastically than $A_{s}$. We attempt to provide an explanation for this. From Eq. 14, we observe that the damping $\Gamma$ depends on the mobility, which obviously reduces with increasing viscosity so that the width of the resonance actually reduces with increasing viscosity as we have demonstrated in Fig. 1(d). How- 
ever, it is clear from Eq. 15, that the resonance frequency changes as $\frac{1}{\eta^{2}}$, while the damping changes as $\frac{1}{\eta}$. Thus, for large value of $\eta$, the narrowing of the width is accompanied by an even smaller change of resonance frequency for a given change in $\eta$. The resultant sensitivity of the resonance response - a combination of both factors - is therefore dominated by the small change in the resonance frequency, which makes the sensitivity lower for increasing viscosity. The change in both phase and amplitude sensitivity also appear almost linear for large viscosities, as shown in the inset of Fig. 2(b). It is also clear that both phase and amplitude sensitivities are highest for the lowest stiffness ratio, which means that they have greater dependence on the sharpness of the resonance than the shift in the central frequency itself. Thus one can, in principle, get higher sensitivities for higher viscosity values by choosing lower values of $k_{1}$ and $k_{2}$, but these imply rather weak optical traps. Thus, we need to perform a trade-off between having the best sensitivity and stable optical traps, so that for our experiments we typically choose values of $k_{2}=3 \mu \mathrm{N} / \mathrm{m}$ and $k_{1}=7 \mu \mathrm{N} / \mathrm{m}$, which result in a viscosity sensitivity of around $0.43 \mathrm{~nm} / 1 \mathrm{e}-4$ Pa.s for $A_{s}$ and $5.3 \mathrm{deg} / 1 \mathrm{e}-4$ Pa.s for $\Phi_{s}$ around the viscosity value of water. Note that these were the stiffness values where we obtained largest $A_{s}$ in Fig. 2(a). We also keep $k_{2}<k_{1}$ for two reasons: 1) To increase the amplitude detection sensitivity of the probe, and 2) to minimize the effect of the probe on the control, i.e. to reduce hydrodynamic back-flow effects.

We now present our experimental setup and results of the measurements of viscosity of different viscous liquids.

\section{EXPERIMENTAL SETUP}

We used a high NA immersion-oil microscope objective (Zeiss PlanApo $100 \times 1.4$ ) to focus two orthogonally polarized laser beams of wavelength $\lambda=1064 \mathrm{~nm}$ to create a dual beam optical tweezers (fig : 3). We employed two different $\frac{\lambda}{2}$ plates to render the polarization state of the two laser beams orthogonal to each other, and an acousto-optic modulator (AOM) to sinusoidally drive one of the traps (driving particle). We used the lens pair L1-L2 to set up a telescopic arrangement to ensure that the AOM was placed at a location that was conjugate to the back focal plane of the microscope objective to exactly project the angular deflection due to the AOM at the back focal plane of the objective lens. To keep the intensity constant (within 1\%), we scanned the first order diffracted beam from the AOM by a very small amount resulting in a very small angular deflection that was magnified at the trap focal plane by employing a long optical path to the microscope objective. As demanded by our measurement scheme, we maintained the other trap stationary. Note that the trap positions along

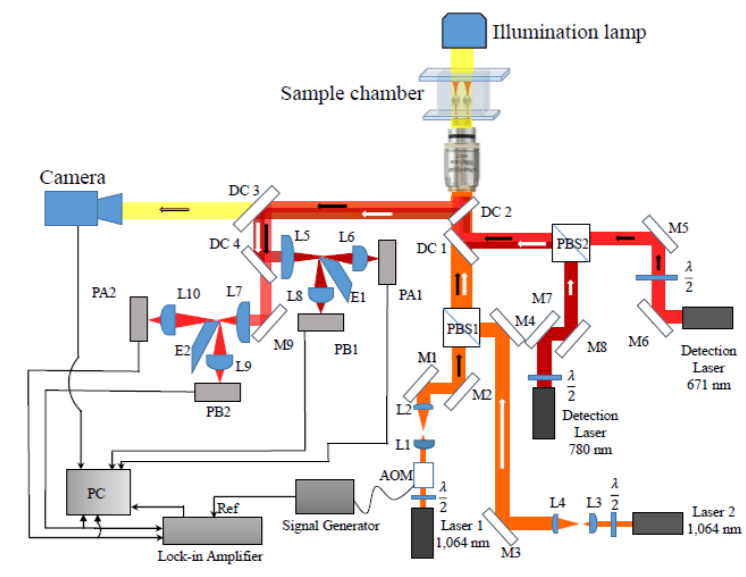

Figure 3. Schematic of the experimental setup. $\frac{\lambda}{2}$ : half wave plate, L: lens, M: mirror, AOM: accousto optical modulator, PBS: polarizing beam splitter, DC: dichroic mirror, E: edge mirror, P: polariser PA, PB: photodiodes (Thorlabs PDA100A-EC), PC: Personal Computer.

the z-axis can be controlled using the lens combination L3 and L4. For our experiment to yield correct results, it is essential that we trap the two particles in the same z-plane. Our control over particle positions along the zaxis is shown in Figure 4(a) and (b). In (a), the particles are not in the same z-plane, as a result of which they appear to be of different size on the image obtained by the microscope camera. In (b), the images are identical, which shows two positions of the two particles on the same z-axis. We prepared two periscope systems by two sets of mirrors (M1, M2 and M3, M4) to independently steer each beam in the $\mathrm{x}-\mathrm{y}$ plane. Later, we used a polarizing beam splitter (PBS1) to recombine the two trapping beams. The detection lasers were of wavelengths $\lambda=671$ and $\lambda=780 \mathrm{~nm}$, and were also prepared to have orthogonal polarization to each other. They were combined by the polarizing beam splitter PBS2. The detection laser beams were overlapped with the trapping laser beams by a dichroic beam splitter (DC1). To detect the positions of the trapped particles by the back-focalplane-interferometry technique, and to image them using white light from the microscope illumination lamp, the back scattered light of the detection lasers and the white light were reflected by the dichroic beam-splitter DC2 towards the detection system and camera, respectively, after being filtered by a third dichroic beam-splitter DC3. The back-scattered detection beams (of different wavelengths as mentioned earlier) were finally separated by the dichroic beam-splitter DC4. The separated detection beams were then focused by two lenses L5 and L7, and balanced detection systems were set up by dividing each beam half-way by edge mirrors E1 and E2, respectively, and focusing them using lenses L6, L8, L10, L9 on photo diode pairs PA1 + PB1 and PA2 + PB2, respectively. We prepared a sealed sample chamber by attaching two 

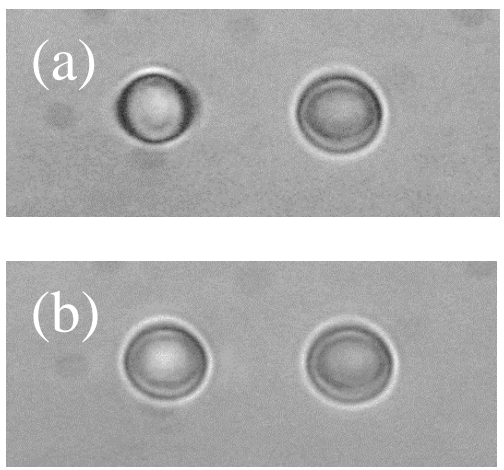

Figure 4. (a) The control and probe particles trapped in different $\mathrm{z}$-planes, which is apparent from the different sizes. (b) The particles trapped in the same plane.

microscope cover slips by double-sided tape so that the dimensions were $20 \times 10 \times 0.2 \mathrm{~mm}$. We used samples of very low volume fraction $(\phi \approx 0.01)$ with $3 \mu \mathrm{m}$ diameter polystyrene spherical particles suspended in a host liquid (water or water + glyercol) constituting the sample. We trapped two such polystyrene particles in two calibrated optical traps (dual optical tweezers) separated by distances varying from 4.5 to $9 \mu \mathrm{m}$, and situated approximately $30 \mu \mathrm{m}$ away from the nearest wall. We generated sinusoidal voltage waveforms from a signal generator (Tektronix, AFG3022B) which were coupled to the AOM to modulate the driving trap. The same we also used as the reference signal of a commercial lockin amplifier (Stanford Research, SR830). We input the detection signals from the balanced detector system for the control particle into the lock-in amplifier to measure amplitude and phase response of the driven particle, and into a computer to calibrate the trap. We used a low pass filter time constant of around $2 \mathrm{~m}$ in the lock-in for each measurement. For the viscosity measurements of the water and glycerol solutions, we prepared different $\mathrm{v} / \mathrm{v}$ mixtures of glycerol and water and measured the corresponding viscosities in a commercial rheometer (Brookfield DB3TLVCJ0) to compare with the measurements obtained using our method.

\section{EXPERIMENTAL RESULTS AND DISCUSSIONS}

To calibrate the individual optical traps independently, we recorded the thermal fluctuations of both driving/driven particles individually with the adjacent trap empty (the corresponding trapping beam was, however, still on). We used the equipartition theorem to measure the stiffness of the trapped single particle. It is important to note that the equipartition method of trap calibration is independent of the rheological nature of the medium. However, careful sensitivity measurements of the detec-
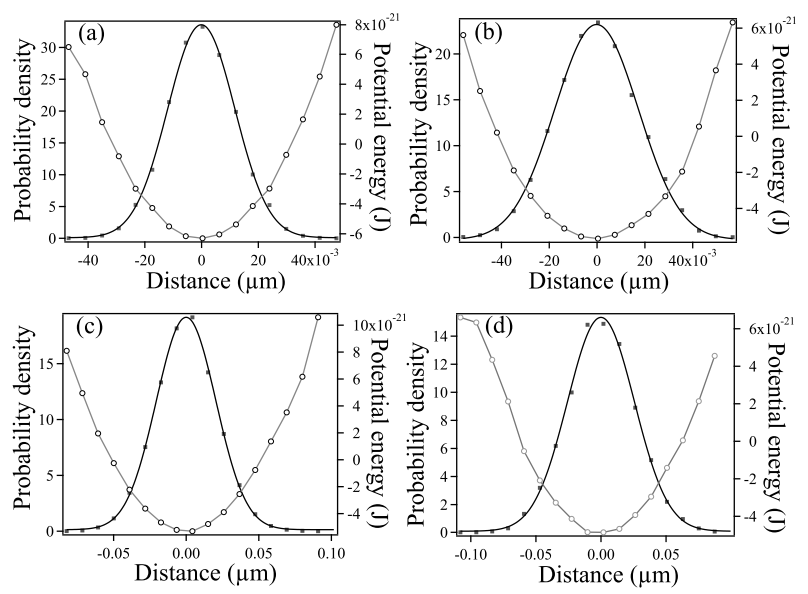

Figure 5. (a) and (c) show the position probability density function plotted against particle displacement for the control particle for trap separations of 9 and $4 \mu m$, respectively, while (b) and (d) show that for the probe particle for the same separations. The solid circles in black are the probability values computed from experimentally measured data, which are then fit Gaussian functions (solid black lines). Each figure also shows the corresponding trap potentials calculated from the same experimental data (right axis, open grey circles), which are fit to quadratic functions of the displacement (solid gray lines).

tion systems are required to calibrate our system. This we performed by shifting the center of the trap potential by a known amount using the AOM and by measuring the corresponding change in the signal from the balanced detection system. Since the potential experienced by the trapped particle is equivalent to that by a harmonic oscillator, the position probability need necessarily be Gaussian, as demanded by the solution of the steady state Fokker-Planck equation $\mathcal{N}\left(0, \frac{k_{B} T}{k}\right)$. This probability distribution thus reflects the harmonic nature of the trap which should be independent of the separation between the two trapped particles. This entails that the trapping potentials should remain Gaussian throughout our experiment. In Fig. 5, we demonstrate that the potential experienced by both the control and probe particles remain Gaussian for the smallest and largest trap separations (4.5 and $9 \mu m$, respectively). This conclusively demonstrates that there exists no optical cross talk between the adjacent traps.

For viscosity measurements on the dual-trap system,

Table I. Measured viscosity of water using phase $\left(\eta_{p}\right)$ and amplitude $\left(\eta_{a}\right)$ for different particle separations.

\begin{tabular}{|c|c|c|}
\hline Separation $(\mu \mathrm{m})$ & $\eta_{p}(\mathrm{~Pa} . \mathrm{s})$ & $\eta_{a}(\mathrm{~Pa}-\mathrm{s})$ \\
\hline 4.5 & $0.00086 \pm 4 e-05$ & $0.0009 \pm 2 e-04$ \\
\hline 7.0 & $0.0009 \pm 5 e-05$ & $0.0011 \pm 1 e-04$ \\
\hline 9.0 & $0.00085 \pm 2.5 e-05$ & $0.00074 \pm 1 e-04$ \\
\hline
\end{tabular}




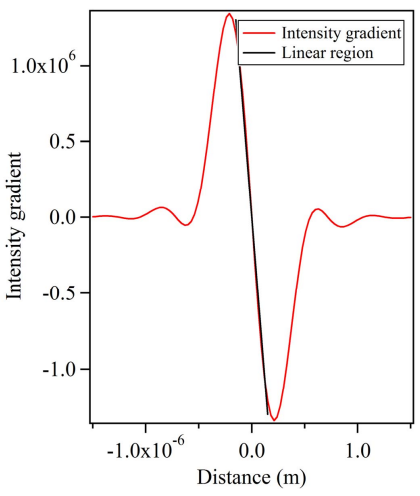

Figure 6. Simulated intensity gradient using our trap parameters. The force acting on the trapped particle is proportional to the intensity gradient - the linear region of which appears to be about $0.3 \mu \mathrm{m}$ from a straight line fit around the center.

we first calibrated each trap individually, and proceeded to measure the response of the driven particle under the influence of the driving particle. As mentioned in Section [II. we chose the driving trap stiffness greater than that of the driven trap (actual values used are provided in Table II). The oscillation amplitude of the control was very small ( $0.25 \mu m$ peak to peak) compared to the actual separation between the two particles, we ensured that the total particle amplitude was maintained within the linear region of the trap. To find out an approximate value of the linear region for our trap, we performed a simple simulation of the potential given our trapping beam parameters as shown in Fig. 6, where we plot the field gradient against distance, and a linear fit near the center shows that the extent of the linear region is around $0.3 \mu \mathrm{m}$, which is larger than the driving beam amplitude. As mentioned earlier, the detection signal from the probe was fed into a lock-in amplifier and the amplitude and phase with respect to the drive were measured at different drive frequencies. Thus, we determined both the zero-crossing of the phase as well as the amplitude maximum of the probe as a function of the drive frequency. While we perform both phase and amplitude measurements, it is obvious that the phase measurement is more reliable, since obtaining sub-nm detection sensitivities as would be needed for measuring viscosity from the amplitude shift - is indeed rather challenging.

Our next step was to determine the relative sensitivity of the amplitude and phase measurement approaches. A reliable method towards achieving this is to measure the respective responses as a function of particle separation, since the hydrodynamic coupling reduces with increasing distance. We performed measurements at three different particle separations: $4.5,7$, and $9 \mu \mathrm{m}$. Our aim was to determine the viscosity of water from both amplitude and phase responses, since the standard value of viscosity of water is very well-known (8.5 e-4 Pa-s at $300 \mathrm{~K})$. Thus, we

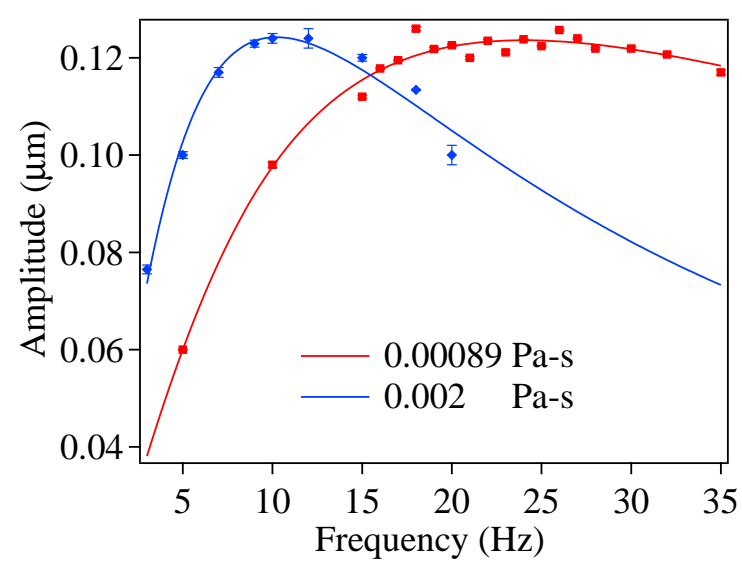

Figure 7. Experimentally measured amplitude responses of the driven particle for two different viscosity solutions. The experimentally measured data are shown in red (blue) solid circles for viscosities of 0.00089 (red) and 0.002 (blue). The data is fit to the amplitude response given in Eq, 10 .

found that the zero-crossing of the phase is more accurate in determining the viscosity reliably, as is shown in Table [1. The value of viscosity of water as obtained from the zero-crossing of the phase is both more accurate (within $2 \%$ ) and precise (statistical error between 3-7\%) compared to the amplitude response - both corresponding errors being almost double that compared to the phase response. The amplitude response also becomes clearly less reliable as the particle separation is increased, which is due to the fact that the signal to noise of the measured data approaches the dark noise levels of the detector as the hydrodynamic coupling reduces.

Finally, we measured the viscosity of different water + glycerol solutions from the phase response of the resonance frequency for each solution. The results are shown in Table II] where $\eta$ is the viscosity measured in the commerical rheometer, and $\eta^{*}$ is the viscosity measured using our resonance technique. The trap stiffnesses were kept the same for all measurements, and the corresponding resonance frequencies $\left(\omega_{0} / 2 \pi\right)$ shifted as shown in Table III. The amplitude response of the resonance for the highest and lowest viscosity solutions are demonstrated in Fig. 7. The value of the resonance frequencies are determined at $3-5 \%$ precision, and the centers shift towards lower frequency with increasing viscosity as predicted by the theory. The measured values of viscosity are all within $2 \%$ of the rheometer measured values. We also note that the accuracy of the measured values we report now have been improved by around 2 times over the measurements we recently reported in Ref. [19], where we performed passive microrheology by using a Bayesian analysis of the position autocorrelation of a single trapped Brownian particle to determine the viscosity of an unknown solution. It needs to be mentioned here that both accuracy and precision can indeed be im- 
Table II. Measured viscosity $\left(\eta^{*}\right)$ compared to that measured in rheometer $(\eta)$. The corresponding trap stiffnesses are also shown.

\begin{tabular}{|c|c|c|c|c|}
\hline \multirow{2}{*}{$\eta($ Pa.S $)$} & \multicolumn{2}{|c|}{ Stiffness $(\mathrm{N} / \mathrm{m})$} & Resonance frequency $(\mathrm{Hz})$ & \multirow{2}{*}{$\eta^{*}(\mathrm{~Pa} . \mathrm{S})$} \\
\cline { 2 - 5 } & $k_{1}$ & $k_{2}$ & $\omega_{0} / 2 \pi$ & \\
\hline 0.00089 & $6.9(1) \mathrm{e}-06$ & $2.7(1) \mathrm{e}-06$ & $24.0 \pm 1.4$ & $0.00088(5)$ \\
\hline 0.00137 & $6.9(2) \mathrm{e}-06$ & $2.7(1) \mathrm{e}-06$ & $15.4 \pm 0.6$ & $0.00138(5)$ \\
\hline 0.0015 & $6.9(1) \mathrm{e}-06$ & $2.7(2) \mathrm{e}-06$ & $14.0 \pm 0.4$ & $0.00151(4)$ \\
\hline 0.001827 & $6.8(1) \mathrm{e}-06$ & $2.8(1) \mathrm{e}-06$ & $11.5 \pm 0.6$ & $0.00184(9)$ \\
\hline 0.0020 & $6.9(3) \mathrm{e}-06$ & $2.8(2) \mathrm{e}-06$ & $10.2 \pm 0.4$ & $0.00204(5)$ \\
\hline
\end{tabular}

proved by using even low stiffness ratios, which would

\section{CONCLUSIONS}

In conclusion, we demonstrate a two-point active rheology technique based on dual trap optical tweezers, where we measure the response of a stationary (probe) colloidal particle when the control particle is driven sinusoidally. The hydrodynamic interactions between control and probe lead to a motional resonance of the probe at a particular driving frequency of the control particle. The resonance frequency is dependent on the stiffnesses of the two traps and the viscosity of the solution where the particles are embedded. The resonance frequency can thus be tuned by changing the trap stiffnesses and liquid viscosity, and increases with the (square root of) product of the two stiffnesses, while reducing with increasing viscosity. Thus, the amplitude and phase responses of the resonance can be used to determine the viscosity if the trap stiffnesses are known. We explicitly determine the analytical expressions for the sensitivity of the phase and amplitude responses with changing trap stiffness ratio as well as liquid viscosity, and observe that the responses are not very sensitive to trap stiffness ratio, but reduce with increasing liquid viscosity. We demonstrate theoretically and perform careful experiments to prove that the phase response has greater accuracy and precision in viscosity measurement compared to the amplitude by measuring the viscosity of water for different separations of the control and probe particles. We then proceed to determine the viscosity of different water and glycerol mixtures, and on comparing our results to that obtained on the same yield sharper resonances and correspondingly larger amplitude and phase sensitivities.

samples by a commerical rheometer, observe agreement to within $2 \%$. This is an improvement of around $2 \mathrm{x}$ in accuracy over our recently reported results in Ref. [19], where we had demonstrated a one-point passive measurement of viscosity by a Bayesian analysis of the Brownian motion trajectories of a trapped particle. This method can be extended to n-particle active microrheology measurements in viscous fluids by holographic tweezers where different sets of control and probe particles can be used and the data collected simultaneously using fast cameras. More interestingly, the resonance characteristics of the probe in a viscoelastic medium should provide even more fascinating results, given that the hydrodynamic interactions in these cases are retarded. This should set up a new paradigm in the active microrheology of complex fluids using optical tweezers - the present set of experiments can be thought of as forming a baseline. We have commenced work in these areas and should be able to report new results in the near future.

\section{ACKNOWLEDGMENTS}

This work was supported by the Indian Institute of Science Education and Research, Kolkata, an autonomous research and teaching institute funded by the Ministry of Human Resource Development, Govt. of India. We acknowledge Dr. Ronojoy Adhikari, Mr. Abhrajit Laskar, and Mr. Rajesh Singh of The Institute of Mathematical Sciences for help in developing the theoretical formalism. The authors also acknowledge Mr. Chayan Dey and 
Dr. Prasun Mondal of Department of Chemical Sciences, IISER Kolkata for their help in the rheometer measurements.

* ayan@iiserkol.ac.in

[1] S. Frases, B. Pontes, L. Nimrichter, M. L. Rodrigues, N. B. Viana, and A. Casadevall, Biophysical J. 97, 937 (2009).

[2] E. J. Robertson, G. Najjuka, M. A. Rolfes, A. Akampurira, N. Jain, J. Anantharanjit, M. von Hohenberg, M. Tassieri, A. Carlsson, D. B. Meya, et al., J. Infect. Dis. 209, 74 (2013).

[3] F. Watts, L. E. Tan, C. G. Wilson, J. M. Girkin, M. Tassieri, and A. J. Wright, J. Opt. 16, 015301 (2013).

[4] J.-P. Hansen and I. R. McDonald, Theory of simple liquids (Academic Press, London, U.K, 1986).

[5] T. G. Mason and D. A. Weitz, Phys. Rev. Lett. 74, 1250 (1995).

[6] T. G. Mason, K. Ganesan, J. H. Van Zanten, D. Wirtz, and S. C. Kuo, Phys. Rev. Lett. 79, 3282 (1997).

[7] F. C. MacKintosh and C. F. Schmidt, Curr. Opin. Coll. Interf. Sc. 4, 300 (1999).

[8] D. Wirtz, Annu. Rev. Biophys. 38, 301 (2009).

[9] T. M. Squires and T. G. Mason, Ann. Rev. Fluid Mech. 42 (2010).

[10] H. D. Ou-Yang, Design and applications of Oscillating Optical Tweezers for Direct Measurements of Colloidal Forces (Wiley, New York, USA, 1999).

[11] K. C. Neuman and A. Nagy, Nature Methods 5, 491 (2008).

[12] D. Mizuno, D. A. Head, F. C. MacKintosh, and C. F. Schmidt, Macromolecules 41, 7194 (2008).

[13] R. R. Brau, J. M. Ferrer, H. Lee, C. E. Castro, B. K. Tam, P. B. Tarsa, P. Matsudaira, M. C. Boyce, R. D. Kamm, and M. J. Lang, Journal of Optics A: Pure and Applied Optics 9, S103 (2007).
[14] C.-C. Chiang, M.-T. Wei, Y.-Q. Chen, P.-W. Yen, Y.-C. Huang, J.-Y. Chen, O. Lavastre, H. Guillaume, D. Guillaume, and A. Chiou, Opt. Express 19, 8847 (2011).

[15] T. Neckernuss, L. K. Mertens, I. Martin, T. Paust, M. Beil, and O. Marti, Journal of Physics D: Applied Physics 49, 045401 (2016)

[16] M. Tassieri, F. D. Giudice, E. J. Robertson, N. Jain, B. Fries, R. Wilson, A. Glidle, F. Greco, P. A. Netti, P. L. Maffettone, T. Bicanic, and J. M. Cooper, Scientific Reports 5, 8831 (2015).

[17] M. Tassieri, G. M. Gibson, R. M. L. Evans, A. M. Yao, R. Warren, M. J. Padgett, and J. M. Cooper, Phys. Rev. E 81, 026308 (2010).

[18] M. Tassieri, R. M. L. Evans, R. L. Warren, N. J. Bailey, and J. M. Cooper, New Journal of Physics 14, 115032 (2012)

[19] S. Bera, S. Paul, R. Singh, D. Ghosh, A. Kundu, A. Banerjee, and R. Adhikari, Sci. Rep. 7, 41638 (2017).

[20] E. J. G. Peterman, F. Gittes, and C. F. Schmidt, Biophys. J. 84, 1308 (2003).

[21] J. C. Crocker, M. T. Valentine, E. R. Weeks, T. Gisler, P. D. Kaplan, A. G. Yodh, and D. A. Weitz, Phys. Rev. Lett. 85, 888 (2000).

[22] J. C. Crocker and B. D. Hoffman, Meth. Cell Biol. 83, 141 (2007).

[23] A. J. Levine and T. C. Lubensky, Phys. Rev. Lett. 85, $1774(2000)$.

[24] A. J. Levine and T. C. Lubensky, Phys. Rev. E 65, 011501 (2001).

[25] T. T. Hormel, M. A. Reyer, and R. Parthasarathy, Biophysical J. 109, 732 (2015).

[26] L. Starrs and P. Bartlett, Journal of Physics: Condensed Matter 15, S251 (2003)

[27] L. D. Landau and E. M. Lifshitz, "Theory of elasticity," (1986).

[28] M. Doi and S. F. Edwards, The theory of polymer $d y$ namics, Vol. 73 (Oxford University Press, 1988).

[29] S. Paul, A. Laskar, R. Singh, B. Roy, R. Adhikari, and A. Banerjee, Phys. Rev. E 96, 050102(R) (2017).

[30] C. W. Gardiner, Handbook of stochastic methods, Vol. 3 (Springer Berlin, 1985). 